\title{
Comparison of long-term clinical outcomes of percutaneous coronary intervention for chronic total occlusion between patients with and without diabetes mellitus: a single-center retrospective observational study
}

\author{
Dongliang $\mathrm{Fu}^{1 \#} \wedge$, Haiwei $\mathrm{Li}^{2 \#}$, Tong $\mathrm{Gao}^{3}$, Mengru Liu ${ }^{3}$, Lina Feng ${ }^{4}$, Chunyan $\mathrm{Li}^{1}$, Xiang Xiao ${ }^{1}$, \\ Hong Jiang ${ }^{1}$, Peng Yang ${ }^{1 *}$, Xianlun $\mathrm{Li}^{1 * \wedge}$ \\ ${ }^{1}$ Cardiology Department of Integrated Traditional Chinese and Western Medicine, China-Japan Friendship Hospital, Beijing, China; ${ }^{2}$ Department \\ of Cardiology, Beijing Anzhen Hospital, Capital Medical University, Beijing, China; ${ }^{3}$ Graduate School of Peking Union Medical College, Beijing, \\ China; ${ }^{4}$ Peking University China-Japan Friendship School of Clinical Medicine, Beijing, China \\ Contributions: (I) Conception and design: D Fu, X Li; (II) Administrative support: H Jiang, P Yang; (III) Provision of study materials or patients: D \\ Fu, C Li, X Xiao; (IV) Collection and assembly of data: D Fu, H Li; (V) Data analysis and interpretation: T Gao, M Liu; (VI) Manuscript writing: All \\ authors; (VII) Final approval of manuscript: All authors. \\ "These authors contributed equally to this work. \\ *These authors contributed equally to this work. \\ Correspondence to: Peng Yang; Xianlun Li. Cardiology Department of Integrated Traditional Chinese and Western Medicine, China-Japan Friendship \\ Hospital, Beijing, China. Email: doctoryp1898@163.com; 1811210693@bjmu.edu.cn.
}

Background: The prognosis of percutaneous coronary intervention (PCI) for chronic total occlusion (CTO) between patients with diabetes mellitus (DM) and those without DM is unknown. This study aimed to investigate whether DM has adverse effects on CTO PCI patients.

Methods: This single-center retrospective study included consecutive patients who underwent PCI for CTO at the China-Japan Friendship Hospital (Beijing, China) between January 2016 and April 2019. The clinical outcomes during follow-up were compared between patients with DM and those without DM.

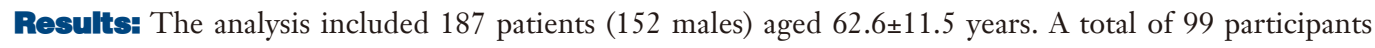
$(52.9 \%)$ had DM, which involved a higher body mass index (BMI) and triglyceride level than those without DM $(\mathrm{P}<0.05)$. Participants with DM and those without DM had similar PCI success rates $(89.9 \%$ vs. $95.4 \%$, respectively) and complete revascularization rates $(82.8 \%$ vs. $84.1 \%$, respectively). There were no significant differences between groups in the rates of all-cause mortality, cardiac death, major adverse cardiovascular events (MACEs), readmission, recurrence of angina, target vessel revascularization (TVR), or myocardial infarction (MI) during a median follow-up of 20.5 months. Multivariable logistic regression revealed that CTO in a coronary branch vessel was associated with higher odds of all-cause death (odds ratio (OR): 53.56; 95\% confidence interval (CI): 2.48 to $1,155.41 ; \mathrm{P}<0.05)$ and failure of PCI for CTO (OR: 5.40; 95\% CI: 1.263 to 23.098; $\mathrm{P}<0.05)$. Additionally, PCI for single CTO was associated with lower odds of MACEs (OR: 0.300; 95\% CI: 0.118 to $0.765 ; \mathrm{P}<0.05)$.

Conclusions: The performance of PCI for CTO has a high success rate in both patients with DM and those without DM, and clinical outcomes are comparable between groups.

Keywords: Ischemic heart disease; chronic total occlusion (CTO); percutaneous coronary intervention (PCI); diabetes; treatment outcome; mortality

^ ORCID: Xianlun Li, 0000-0002-2460-2255; Dongliang Fu, 0000-0003-3003-4255. 
Submitted Jul 23, 2021. Accepted for publication Sep 10, 2021.

doi: 10.21037/apm-21-2354

View this article at: https://dx.doi.org/10.21037/apm-21-2354

\section{Introduction}

Ischemic heart disease is a major cause of morbidity and hospitalization worldwide and is responsible for around 9 million deaths each year (1). The prevalence of ischemic heart disease has increased rapidly over recent years as a result of population aging and lifestyle changes, and China has seen a large increase in hospital admissions for acute coronary syndrome during the last decade $(2,3)$. Various options are available for the management of coronary heart disease (CHD) including revascularization procedures such as coronary artery bypass grafting $(\mathrm{CABG})$ and percutaneous coronary intervention (PCI) (4).

The degree of stenosis of a coronary artery is an important indicator of CHD severity that plays a key role in treatment decision making. Chronic total occlusion (CTO) is observed in around $30-50 \%$ of patients undergoing coronary angiography (5), and until recently was managed preferentially with CABG rather than PCI due to the perceived limited success rate of PCI. Although PCI for CTO has had a steep learning curve (6), it is now recognized that the procedural success rate of $\mathrm{PCI}$ can reach around $90 \%$ in experienced centers (7-9). Furthermore, several studies have shown that successful PCI for CTO is associated with better clinical outcomes than failed PCI (9-11).

Diabetes mellitus (DM) is a well-known risk factor for cardiovascular morbidity and mortality (12). It is a highly prevalent chronic disease affecting more than 420 million adults globally, and it is predicted that the number of people affected by DM will increase to 642 million by 2040 (13). The prevalence of DM has been progressively increasing in China, where the age-standardized prevalence of DM and pre-diabetes is estimated to be $9.7-11.6 \%(14,15)$. Notably, recent epidemiological surveys have reported that about $27-45 \%$ of patients with CTO have DM $(16,17)$.

The optimal treatment strategy for patients with CTO and DM deserves particular attention due to the treatment-risk paradox and the absence of evidence-based recommendations (18). Clinical studies have suggested that PCI, when performed at high-volume centers by highly experienced operators, is a safe and effective treatment that can provide complete myocardial revascularization in selected patients with CTO and DM $(19,20)$. Nevertheless, there are limited published data evaluating whether DM influences the outcomes of PCI for CTO. Therefore, the aim of this study was to compare the long-term clinical outcomes of PCI for CTO between patients with DM and those without DM. We present the following article in accordance with the STROBE reporting checklist (available at https://dx.doi.org/10.21037/apm-21-2354).

\section{Methods}

\section{Study design and patients}

This single-center retrospective study included patients who underwent PCI for CTO at the Cardiology Department, Integrated Traditional Chinese and Western Medicine, China-Japan Friendship Hospital (Beijing, China) between January 2016 and April 2019. The eligibility criteria included the presence of at least 1 CTO in a principal coronary artery and revascularization with PCI. The exclusion criteria were: (I) presence of severe coagulation abnormalities, malignant tumor with a life expectancy shorter than 1 year, or other end-stage disease; (II) incomplete medical history or follow-up data; and (III) contraindications to coronary angiography. The study was approved by the Ethics Committee of the China-Japan Friendship Hospital (No. 2020-14-K11) and complied with the principles outlined in the Declaration of Helsinki (as revised in 2013). All participants signed informed consent documents for the procedure itself and the use of their anonymized data in research.

\section{Definitions}

We defined CTO was as angiographic evidence of thrombolysis in myocardial infarction (TIMI) flow grade 0 within an occluded arterial segment for more than 3 months (21). We defined DM as current therapy with oral hypoglycemic drugs or insulin, fasting plasma glucose $\geq 7.0 \mathrm{mmol} / \mathrm{L}(126 \mathrm{mg} / \mathrm{dL})$, or 2 -hour plasma glucose $\geq 11.1 \mathrm{mmol} / \mathrm{L}(200 \mathrm{mg} / \mathrm{dL})$ after a standard $75 \mathrm{~g}$ oral glucose tolerance test (22). Successful PCI for CTO was defined as the recanalization of the lesion with a residual stenosis level below $30 \%$ and a TIMI flow grade 3 (21). Complete revascularization was defined by the operators 
as successful treatment of all physiologically significant coronary stenosis (23). Target vessel revascularization (TVR) was defined as any repeat PCI of the target vessel performed for restenosis or any other complication of the target lesion. Chronic kidney disease (CKD) was defined as an estimated glomerular filtration rate $<60 \mathrm{~mL} / \mathrm{min} / 1.73 \mathrm{~m}^{2}$ for at least 3 months or chronic dialysis.

\section{PCI procedure}

The PCI and stent implantation were carried using standard contemporary techniques such as bilateral injection, the hybrid algorithm, tapered-tip guidewires, stiff wires, parallel wires, microcatheters, and the retrograde approach as these became available. Drug-eluting stents were implanted in successful procedures. Following PCI, all participants were prescribed clopidogrel for least 12 months and lifelong aspirin.

\section{Data collection}

Clinical and laboratory data were extracted from the medical records by independent research personnel. The clinical data included baseline information [such as gender, age, body mass index (BMI), and CTO course], clinical history (smoking status, hypertension, CKD, heart failure, peripheral vascular disease, and cerebrovascular disease), symptom evaluation [Canadian Cardiovascular Society (CCS) angina severity classification], other relevant medical information, details of the surgical procedure (such as CTO length, lesion length in the target vessel, total stent length, number of vessels with CTO, CTO location, and PCI success/failure), and postoperative follow-up data.

\section{Follow-up and clinical outcomes}

Participants were followed-up by telephone interview, outpatient visit, or physician contact from the day of surgery to 1 April 2020. Those lost to very long-term follow-up were censored at the time of their last contact. The outcomes included all-cause death (death during follow-up due to any reason), major adverse cardiovascular events [MACEs, defined as a composite of cardiac death, myocardial infarction (MI) and TVR], readmission (rehospitalization due to cardiovascular-related diseases), and recurrent angina (the recurrence or worsening of angina after surgery).

\section{Statistical analysis}

For the analysis, participants were divided into 2 groups according to the presence of DM. Missing data analysis procedures were used to directly delete missing values. Normally-distributed continuous data were presented as the mean \pm standard deviation (SD) and were compared between groups using Student's $t$-test. Non-normally distributed continuous data were described as median and interquartile range (IQR) and were compared between groups using the Wilcoxon rank-sum test. Categorical data were presented as frequencies or percentages and compared between groups using the chi-squared test or Fisher's exact test, as appropriate. A 2 -sided $\mathrm{P}$ value $<0.05$ was considered statistically significant. A multivariable model was built by stepwise variable selection with entry and exit criteria set at the $\mathrm{P} \leq 0.1$ level, and odds ratios (OR), $95 \%$ confidence intervals $(95 \% \mathrm{CI})$ were calculated. All statistical analyses were performed using SPSS 20.0 (IBM Corp., Armonk, NY, USA).

\section{Results}

\section{Baseline clinical characteristics of participants}

A total of 187 consecutive patients undergoing PCI for CTO were enrolled during the study period. The 187 participants had a mean age of $62.6 \pm 11.5$ years (152 males aged $60 \pm 11$ years and 35 females aged $72 \pm 8$ years). A total of 99 participants $(52.9 \%)$ had DM. The baseline clinical characteristics of participants are shown in Table 1. Participants with DM had significantly higher BMI, fasting blood glucose level, glycated hemoglobin (HbA1c) level, glycated albumin (GA) level, triglyceride (TG) level, and use of angiotensin receptor blockers than those without $\mathrm{DM}(\mathrm{P}<0.05$ for all parameters; Table 1$)$. Note that, for this analysis, a total of 19 patients $(10.2 \%)$ were lost to followup. There was no significant difference between the DM group and non-DM group in the number of patients lost to follow-up (14 vs. 5 cases; $\mathrm{P}=0.095$ ).

\section{Angiographic and procedural characteristics}

Table 2 summarizes the angiographic and procedural characteristics. The majority of participants had multivessel disease, and this was more common in those with DM than in those without $\mathrm{DM}(85.9 \%$ vs. $71.6 \%, \mathrm{P}<0.05)$. Lesions in the lateral circumflex artery (LCX) and CTO of the 
Table 1 Baseline clinical characteristics of the participants stratified according to DM status

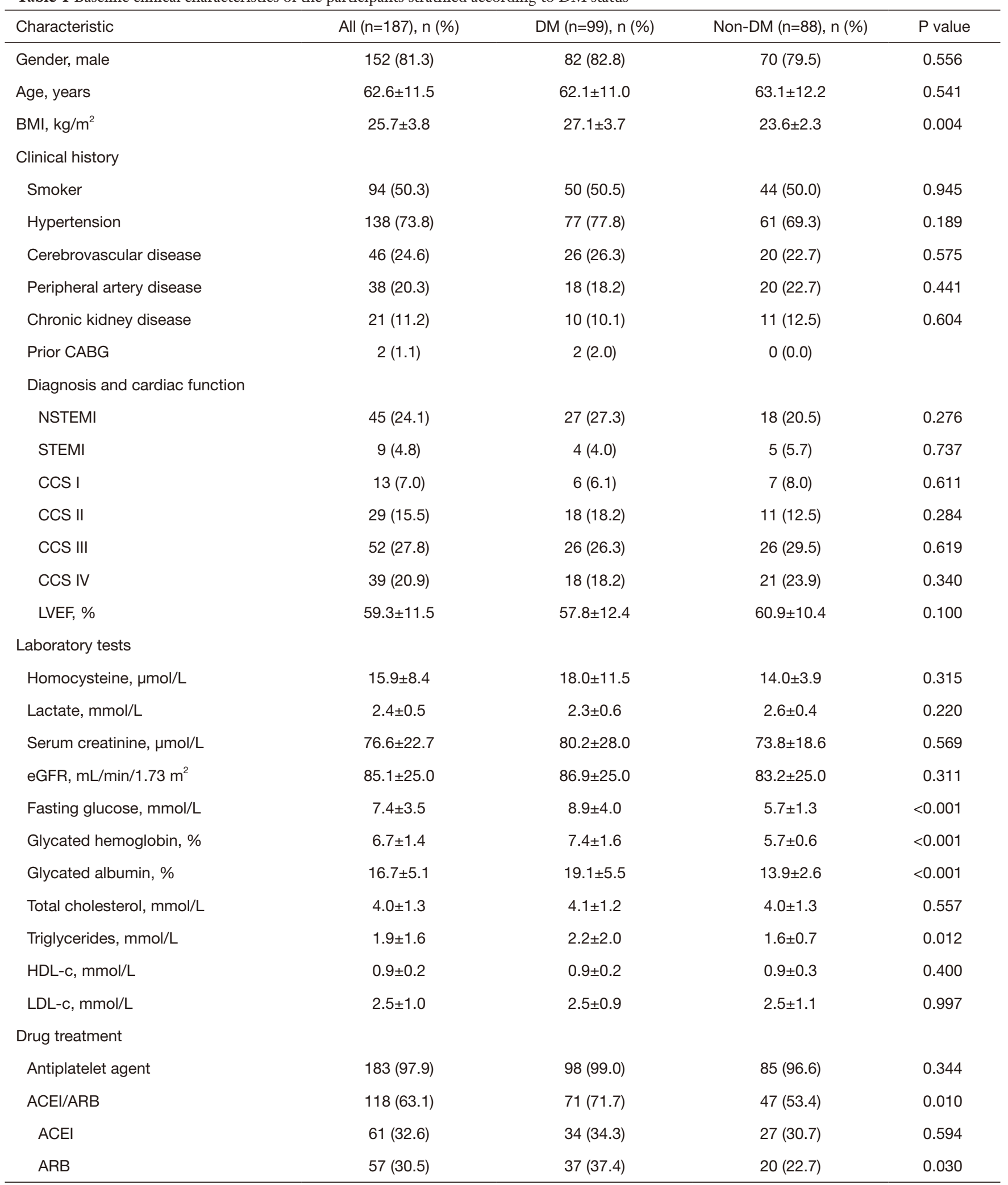

Table 1 (continued) 
Table 1 (continued)

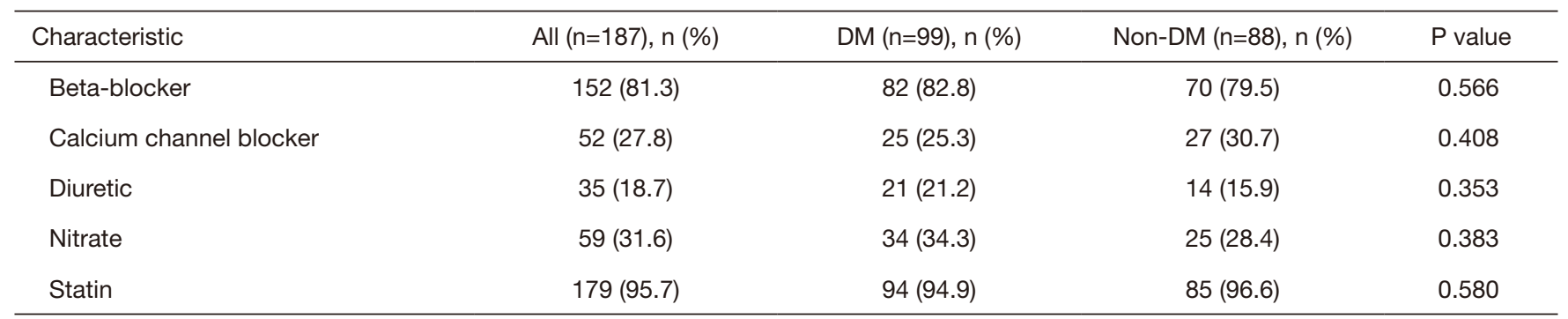

Data are presented as mean \pm standard deviation or $\mathrm{n}(\%)$. DM, diabetes mellitus; BMI, body mass index; ACEI, angiotensin-converting enzyme inhibitors; ARB, angiotensin receptor blocker; CABG, coronary artery bypass graft; CCS, Canadian Cardiovascular Society; eGFR, estimated glomerular filtration rate; HDL-c, high density lipoprotein cholesterol; LDL-c, low density lipoprotein cholesterol; LVEF, left ventricular ejection fraction; NSTEMI, non-ST segment elevation myocardial infarction; STEMI, ST segment elevation myocardial infarction.

LCX were more common in participants with DM than in those without $\mathrm{DM}(\mathrm{P}<0.05$ for both parameters; Table 2). All other angiographic features were comparable between groups (Table 2). The complete revascularization rate was similar for participants with DM $(82.8 \%)$ and those without DM (84.1\%). The overall success rate of PCI for CTO was 92.5\%. Furthermore, the success rate of PCI for CTO did not differ significantly between participants with DM and those without DM (89.9\% and $95.4 \%$, respectively).

\section{Clinical outcomes}

A total of 4 participants $(2.1 \%)$ died during a median follow-up time of 20.5 months (IQR, 13.0-27.8 months). Table 3 compares the clinical outcomes between participants with DM and those without DM.

\section{Subgroup analyses}

Subgroup analyses of participants in the DM and non-DM groups were carried out to determine whether hypertension, $\mathrm{CKD}$, and incomplete revascularization were factors associated with clinical outcomes (Table 4). Hypertension was not associated with any of the clinical outcomes in either the DM or non-DM group. However, CKD was associated with a significantly higher incidence of all-cause death, MACEs, readmission, and recurrence of angina in participants without $\mathrm{DM}(\mathrm{P}<0.05$ for all parameters; Table 4). Additionally, incomplete revascularization was associated with a significantly higher incidence of TVR, MI, readmission and recurrence of angina in patients without $\mathrm{DM}(\mathrm{P}<0.05$ for all parameters; Table 4$)$.

\section{Analysis of factors associated with clinical outcomes}

The results of the univariable and multivariable regression analyses are detailed in Table 5. Multivariable analyses revealed that CTO in a branch vessel was independently associated with higher odds of all-cause death (OR: 53.56; 95\% CI: 2.48 to $1,155.41 ; \mathrm{P}<0.05)$ and higher odds of failure of PCI for CTO (OR: 5.40; 95\% CI: 1.263 to 23.098; $\mathrm{P}<0.05)$. Furthermore, PCI for single CTO was independently associated with lower odds of MACEs (OR: 0.300; $95 \%$ CI: 0.118 to 0.765 ; $\mathrm{P}<0.05)$.

\section{Discussion}

The main findings of this study were as follows: (I) among participants with CTO who underwent PCI, those with DM had a higher BMI and triglyceride level and more common use of angiotensin receptor blockers (which would be in accordance with current guidelines) than those without DM; (II) multiple coronary artery disease was common in participants with CTO and DM, with the LCX frequently involved; (III) there were no significant differences in clinical outcomes between patients with DM and those without DM; (IV) CTO of a branch vessel was an independent predictor of cardiac mortality and PCI failure for CTO, while successful treatment of single CTO with PCI was associated with a reduced incidence of MACEs; and $(V)$ in participants without DM, CKD was associated with a worse prognosis. Taken together, our findings demonstrate that PCI has a high success rate in the management of CTO in both patients with DM and those without DM. Furthermore, clinical outcomes after PCI for 
Table 2 Angiographical and procedural characteristics stratified according to DM status

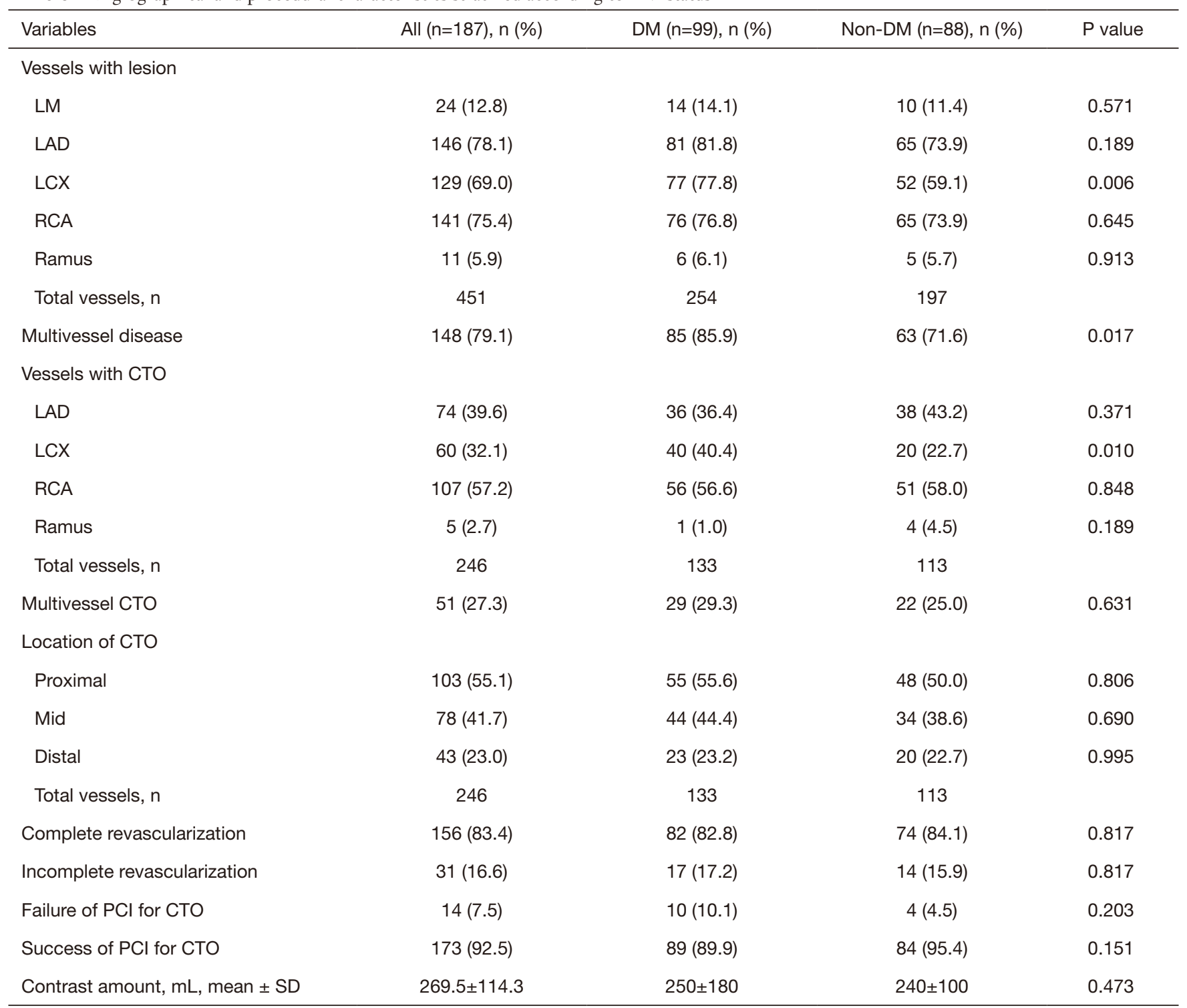

Data are presented as mean \pm standard deviation or $\mathrm{n}$ (\%). CTO, chronic total occlusion; DM, diabetes mellitus; LAD, left anterior descending artery; LCX, left circumflex artery; LM, left main artery; $\mathrm{PCl}$, percutaneous coronary intervention; RCA, right coronary artery; $\mathrm{SD}$, standard deviation.

CTO are comparable between patients with DM and those without DM.

In the present study, $52.9 \%(99 / 187)$ of participants had $\mathrm{DM}$, while previous research has indicated that $27-45 \%$ of patients with CTO have DM $(16,17,24)$. The high prevalence of DM among patients with CTO is consistent with the finding that patients with DM tend to have more severe and widespread coronary atherosclerosis than those without DM (25). Additionally, our cohort contained far more males $(81.3 \%)$ than females $(18.7 \%)$, which is consistent with previous studies of patients with CTO $(26,27)$. Interestingly, the female participants were on average 10 years older than the male patients, which might reflect a delay in the disease process in women due to them being at lower risk of coronary atherosclerotic disease before menopause. It is also recognized that women with ischemic heart disease are more likely to receive medical treatment and less likely to undergo PCI than men (28). We also 
Table 3 Clinical outcomes for patients with DM and those without DM

\begin{tabular}{|c|c|c|c|c|}
\hline Parameter & All $(n=187), n(\%)$ & $\mathrm{DM}(\mathrm{n}=99), \mathrm{n}(\%)$ & Non-DM (n=88), n (\%) & $P$ value \\
\hline All-cause death & $4(2.4)$ & $1(1.2)$ & $3(3.6)$ & 0.300 \\
\hline Cardiac death & $1(0.6)$ & $0(0.0)$ & $1(1.2)$ & \\
\hline MACE & $23(13.7)$ & $12(14.1)$ & $11(13.3)$ & 0.871 \\
\hline Target vessel revascularization & $15(8.9)$ & $8(9.4)$ & $7(8.4)$ & 0.824 \\
\hline Readmission & $41(24.4)$ & $18(21.2)$ & $23(27.7)$ & 0.324 \\
\hline Angina recurrence & $42(25.0)$ & $17(20.0)$ & $25(30.1)$ & 0.130 \\
\hline
\end{tabular}

Data are presented as mean \pm standard deviation or $\mathrm{n}(\%)$. DM, diabetes mellitus; MACE, major adverse cardiovascular event; SD, standard deviation.

Table 4 Subgroup analyses evaluating hypertension, chronic kidney disease, and incomplete revascularization as possible factors associated with clinical outcomes

\begin{tabular}{|c|c|c|c|c|c|c|c|c|}
\hline Subgroup & Stratification factor & $\begin{array}{l}\text { All-cause } \\
\text { death }\end{array}$ & $\begin{array}{c}\text { Cardiac } \\
\text { death }\end{array}$ & MACE & $\mathrm{Ml}$ & TVR & Readmission & $\begin{array}{l}\text { Angina } \\
\text { recurrence }\end{array}$ \\
\hline \multirow[t]{2}{*}{$\mathrm{DM}(\mathrm{n}=85)$} & HT (n=65), n (\%) & 0 & 0 & $10(15.4)$ & $7(10.8)$ & $8(12.3)$ & $16(24.6)$ & $16(24.6)$ \\
\hline & $P$ value & & & 0.723 & 0.671 & & 0.219 & 0.062 \\
\hline \multirow[t]{2}{*}{ Non-DM (n=83) } & HT $(n=56), n(\%)$ & $2(3.6)$ & $1(1.8)$ & $5(8.9)$ & $2(3.6)$ & $3(5.4)$ & $14(25.0)$ & $17(30.4)$ \\
\hline & $P$ value & 0.976 & & 0.094 & 1 & 0.207 & 0.427 & 0.946 \\
\hline \multirow[t]{2}{*}{$\mathrm{DM}(\mathrm{n}=85)$} & CKD (n=8), n (\%) & 0 & 0 & 0 & 0 & 0 & $2(25.0)$ & $2(25.0)$ \\
\hline & Non-CKD $(n=77), n(\%)$ & $1(1.3)$ & 0 & $12(15.6)$ & $8(10.4)$ & $8(10.4)$ & $16(20.8)$ & $15(19.5)$ \\
\hline Non-DM ( $n=83)$ & $P$ value & 0.023 & & 0.033 & 0.265 & & 0.021 & 0.008 \\
\hline \multirow[t]{3}{*}{$\mathrm{DM}(\mathrm{n}=85)$} & CR $(n=71), n(\%)$ & $1(1.4)$ & 0 & $9(12.7)$ & $5(7.0)$ & $5(7.0)$ & $14(19.7)$ & $14(19.7)$ \\
\hline & Non-CR (n=14), n (\%) & 0 & 0 & $3(21.4)$ & $3(21.4)$ & $3(21.4)$ & $4(28.6)$ & $3(21.4)$ \\
\hline & $P$ value & & & 0.408 & 0.092 & 0.092 & 0.483 & 0.884 \\
\hline \multirow[t]{3}{*}{ Non-DM (n=83) } & CR (n=69), n (\%) & $3(4.3)$ & $1(1.4)$ & 8 (11.6) & $2(2.9)$ & $3(4.3)$ & $15(21.7)$ & $18(26.1)$ \\
\hline & Non-CR $(n=14), n(\%)$ & 0 & 0 & $3(21.4)$ & $1(7.1)$ & $4(28.6)$ & $8(57.1)$ & $7(50.0)$ \\
\hline & $\mathrm{P}$ & & & 0.386 & 0.043 & 0.014 & 0.018 & 0.075 \\
\hline
\end{tabular}

Data are presented as n (\%). CKD, chronic kidney disease; CR, complete revascularization; DM, diabetes mellitus; HT, hypertension; MACE, major adverse cardiovascular event; MI, myocardial infarction; TVR, target vessel revascularization. 
Table 5 Logistic regression analysis of factors associated with clinical outcomes

\begin{tabular}{|c|c|c|c|c|c|c|c|}
\hline Factor & Regression & \multicolumn{2}{|l|}{ All-cause death } & \multicolumn{2}{|l|}{ MACE } & \multicolumn{2}{|c|}{$\mathrm{PCl}$ failure for CTO } \\
\hline \multirow{2}{*}{$\begin{array}{l}\text { CTO in } \\
\text { branch }\end{array}$} & Univariable & $13.333(1.127-157.800)$ & 0.04 & $1.602(0.171-15.004)$ & 0.68 & $7.810(2.020-30.462)$ & 0.003 \\
\hline & Multivariable & $53.56(2.480-1,155.410)$ & 0.011 & $2.173(0.164-28.758)$ & 0.556 & $5.400(1.263-23.098)$ & 0.023 \\
\hline $\begin{array}{l}\text { Success of } \\
\text { single PCl } \\
\text { for CTO }\end{array}$ & Univariable & $1.169(0.119-11.533)$ & 0.893 & $0.360(0.146-0.887)$ & 0.026 & & \\
\hline \multirow[t]{2}{*}{ CKD } & Univariable & $10.714(1.400-81.993)$ & 0.022 & $0.657(0.172-2.509)$ & 0.538 & $0.740(0.154-3.562)$ & 0.707 \\
\hline & Multivariable & $4.181(0.340-51.391)$ & 0.264 & $0.705(0.177-2.808)$ & 0.62 & $0.603(0.094-3.851)$ & 0.593 \\
\hline \multirow[t]{2}{*}{ CCS IV } & Univariable & $4.125(0.560-30.408)$ & 0.164 & $2.973(0.662-13.36)$ & 0.155 & $3.378(1.171-9.750)$ & 0.024 \\
\hline & Multivariable & $4.376(0.326-58.667)$ & 0.265 & $3.927(0.752-20.517)$ & 0.105 & $2.396(0.757-7.577)$ & 0.137 \\
\hline
\end{tabular}

CKD, chronic kidney disease; CCS, Canadian Cardiovascular Society; CTO, chronic total occlusion; MACE, major adverse cardiovascular event; $\mathrm{PCl}$, percutaneous coronary intervention; OR, odds ratio; $\mathrm{Cl}$, confidence interval.

found that patients with DM had a higher level of TG than those without DM, in agreement with research showing that lipid metabolism disorders often co-exist with DM and accelerate the atherosclerotic process (26). In our study, Patients with DM had significantly higher fasting blood glucose levels, which further analysis found no correlation with mortality. However, Sinnaeve et al. demonstrate that fasting blood glucose levels at admission are associated with increased mortality after acute myocardial infarction (AMI), and the increased risk is short-term and 6-month mortality (29).

Diabetes is one important risk factor for CAD. The accelerated atherosclerotic burden and increased CTO lesions complicated observed in diabetic individuals. Compared with non-diabetic individuals, Patients with DM often present with diffuse, small-vessel and multivessel coronary artery disease. The anatomical coronary complexity and metabolic disorders in patients with DM may results in more challenging vessel revascularization and worse long-term prognosis (30). In our study, multivessel disease was significantly more frequent in participants with $\mathrm{DM}$ than in those without DM, which is in agreement with previous research (25). Interestingly, the LCX was more commonly involved in patients with DM. Lesions of the LCX are considered to increase the risk of coronary artery perforation and the difficulty of surgery, and CTO of the LCX has been included in a model predicting the success rate of PCI for CTO (31). Nevertheless, we did not observe any significant differences in complete revascularization rate or PCI success rate between the DM and non-DM groups.
Although a previous report suggested that the complete recanalization rate was lower for patients with DM than for those without DM (26), other studies have described similar angiographic outcomes for the 2 groups (32), which would be consistent with our results. The high recanalization rate observed in our study is an important finding, because successful recanalization is an independent predictor of long-term survival (33-36). The prevalence of concomitant CTO in patients with MI-associated cardiogenic shock is associated with adverse outcomes. Within a CULPRITSHOCK sub-analysis, the presence of a non-infarct related CTO in patients with cardiogenic shock was higher and was associated with a higher rate of death at 30 days and 1 year. while a strategy of culprit-lesion-only PCI seems beneficial regardless of the presence of CTO (37).

Some clinical studies have suggested that patients with CTO and DM are at higher risk of long-term adverse outcomes (such as MACEs, TVR, and death) than those with CTO who do not have DM $(36,38)$. Different from the results of Sanguineti $e$ al., we found that diabetes was not a predictor of cardiac mortality in patients with CTO lesion and CTO recanalisation also cannot reduced cardiac mortality and MACEs among diabetic patients (27). The different results may be come from the development of equipment and technique in recent years. Recently other studies also found no significant difference in the procedural success or safety of CTO PCI and similar improvements in health status following CTO PCI between patients with and without diabetes (26). However, the concept that high-risk patients are less frequently treated than lower 
risk individuals, a so-called 'treatment-risk paradox', is not new in the field of PCI (30). A notable finding of the present study was that the clinical outcomes did not differ significantly between participants with DM and those without DM. Our observations are consistent with those of 2 previous studies $(32,39)$, which implies that patients with CTO and DM can achieve the same benefit from PCI as those without DM. Nevertheless, prospective, randomized studies are needed to directly compare the clinical benefits of PCI for CTO between patients with DM and those without.

Previous studies have reported that revascularization is superior to medical therapy as a treatment for CTO in patients with DM (24) and CKD (40). In our study, CKD was associated with a significantly higher incidence of adverse clinical outcomes including all-cause death, MACEs, readmission, and recurrence of angina. The above finding concurs with prior research indicating that renal insufficiency is an important predictor of mortality after PCI for CTO $(41,42)$. However, additional data are needed to fully establish the relevance of CKD to the outcomes of patients with CTO who are treated using PCI.

Our multivariable regression analysis showed that CTO of a coronary branch was associated with increased odds of PCI failure and all-cause death. The presence of $\mathrm{DM}$ is a risk factor for collateral circulation formation, and branch lesions can reflect the coronary collateral circulation (CCC) to some extent. The quantity of viable myocardium is associated with the CCC, which may be an underlying factor that impacts on left ventricular ejection fraction $(43,44)$. Therefore, preoperative imaging should be performed to evaluate the viable myocardium supplied by the affected artery and screen for patients predicted to respond to revascularization. The treatment of CAD using invasive PCI has evolved dramatically in the last decades. The main evolvement is the update of stents and the application of new techniques. The next major advance in the evolving field of PCI may be the biodegradable polymer stents and bioresorbable vascular scaffolds, although their efficacy, safety and ultimately their place in therapy remain to be determined.

This study had some limitations. First, this study was a retrospective study, so these results may be prone to selection bias or information bias. Second, this was a singlecenter study, so it is not known whether the findings are generalizable, and it needs to be further confirmed by multi-center clinical trials. Third, the sample size was small, so the study may have been underpowered to detect some real differences between groups. Fourth, this observational study did not provide a comparison of outcomes with a medically treated arm. Fifth, the data were collected using the hospital information system and telephone followup, and it is possible that unknown confounding factors in the information gathering process may have affected the results. Additionally, the results of this study may have been influenced by the selection criteria, operator experience, and varying techniques used by the operators.

\section{Conclusions}

Although multiple coronary artery lesions were more common in participants with DM than in those without $\mathrm{DM}$, procedural success rate and clinical outcomes were comparable between the 2 groups. Additionally, coronary branch disease was associated with a higher rate of failure of PCI for CTO.

\section{Acknowledgments}

The authors gratefully acknowledge the cooperation of all participants.

Funding: This work was supported by the National Key Research and Development Program of China (2017YFF0205401).

\section{Footnote}

Reporting Checklist: The authors have completed the STROBE reporting checklist. Available at https://dx.doi. org/10.21037/apm-21-2354

Data Sharing Statement: Available at https://dx.doi. org/10.21037/apm-21-2354

Conflicts of Interest: All authors have completed the ICMJE uniform disclosure form (available at https://dx.doi. org/10.21037/apm-21-2354). The authors have no conflicts of interest to declare.

Ethical Statement: The authors are accountable for all aspects of the work in ensuring that questions related to the accuracy or integrity of any part of the work are appropriately investigated and resolved. The study was approved by the Ethics Committee of the China-Japan Friendship Hospital (No. 2020-14-K11) and complied with the principles outlined in the Declaration of Helsinki (as 
revised in 2013). All participants signed informed consent documents for the procedure itself and for the use of their anonymized data in research.

Open Access Statement: This is an Open Access article distributed in accordance with the Creative Commons Attribution-NonCommercial-NoDerivs 4.0 International License (CC BY-NC-ND 4.0), which permits the noncommercial replication and distribution of the article with the strict proviso that no changes or edits are made and the original work is properly cited (including links to both the formal publication through the relevant DOI and the license). See: https://creativecommons.org/licenses/by-nc-nd/4.0/.

\section{References}

1. GBD 2017 Causes of Death Collaborators. Global, regional, and national age-sex-specific mortality for 282 causes of death in 195 countries and territories, 1980-2017: a systematic analysis for the Global Burden of Disease Study 2017. Lancet 2018;392:1736-88.

2. Fuster V. Changing Demographics: A New Approach to Global Health Care Due to the Aging Population. J Am Coll Cardiol 2017;69:3002-5.

3. Wu Y, Benjamin EJ, MacMahon S. Prevention and Control of Cardiovascular Disease in the Rapidly Changing Economy of China. Circulation 2016;133:2545-60.

4. Kobayashi A, Misumida N. Percutaneous coronary intervention in left main disease: 10-year follow-up. Ann Transl Med 2019;7:85.

5. Holmes DR Jr, Barsness GW. Percutaneous Coronary Intervention for Chronic Total Occlusions. Circ Cardiovasc Interv 2019;12:e008321.

6. Young MN, Secemsky EA, Kaltenbach LA, et al. Examining the Operator Learning Curve for Percutaneous Coronary Intervention of Chronic Total Occlusions. Circ Cardiovasc Interv 2019;12:e007877.

7. Lee SW, Lee PH, Ahn JM, et al. Randomized Trial Evaluating Percutaneous Coronary Intervention for the Treatment of Chronic Total Occlusion. Circulation 2019;139:1674-83.

8. Werner GS, Martin-Yuste V, Hildick-Smith D, et al. A randomized multicentre trial to compare revascularization with optimal medical therapy for the treatment of chronic total coronary occlusions. Eur Heart J 2018;39:2484-93.

9. Mashayekhi K, Nührenberg TG, Toma A, et al. A Randomized Trial to Assess Regional Left Ventricular Function After Stent Implantation in Chronic Total
Occlusion: The REVASC Trial. JACC Cardiovasc Interv 2018;11:1982-91.

10. Mehran R, Claessen BE, Godino C, et al. Long-term outcome of percutaneous coronary intervention for chronic total occlusions. JACC Cardiovasc Interv 2011;4:952-61.

11. Jones DA, Weerackody R, Rathod K, et al. Successful recanalization of chronic total occlusions is associated with improved long-term survival. JACC Cardiovasc Interv 2012;5:380-8.

12. Rosiek A, Leksowski K. The risk factors and prevention of cardiovascular disease: the importance of electrocardiogram in the diagnosis and treatment of acute coronary syndrome. Ther Clin Risk Manag 2016;12:1223-9.

13. Ogurtsova K, da Rocha Fernandes JD, Huang Y, et al. IDF Diabetes Atlas: Global estimates for the prevalence of diabetes for 2015 and 2040. Diabetes Res Clin Pract 2017;128:40-50.

14. Yang W, Lu J, Weng J, et al. Prevalence of diabetes among men and women in China. $\mathrm{N}$ Engl $\mathrm{J}$ Med 2010;362:1090-101.

15. Xu Y, Wang L, He J, et al. Prevalence and control of diabetes in Chinese adults. JAMA 2013;310:948-59.

16. Fefer P, Knudtson ML, Cheema AN, et al. Current perspectives on coronary chronic total occlusions: the Canadian Multicenter Chronic Total Occlusions Registry. J Am Coll Cardiol 2012;59:991-7.

17. Choi KH, Yang JH, Song YB, et al. Long-term clinical outcomes of patients with coronary chronic total occlusion treated with percutaneous coronary intervention versus medical therapy according to presence of diabetes mellitus. EuroIntervention 2017;13:970-7.

18. Roffi M, Angiolillo DJ, Kappetein AP. Current concepts on coronary revascularization in diabetic patients. Eur Heart J 2011;32:2748-57.

19. Rha SW, Choi BG, Baek MJ, et al. Five-Year Outcomes of Successful Percutaneous Coronary Intervention with Drug-Eluting Stents versus Medical Therapy for Chronic Total Occlusions. Yonsei Med J 2018;59:602-10.

20. Flores-Umanzor EJ, Cepas-Guillen PL, Vázquez S, et al. Survival benefit of revascularization versus optimal medical therapy alone for chronic total occlusion management in patients with diabetes. Catheter Cardiovasc Interv 2021;97:376-83.

21. Stone GW, Kandzari DE, Mehran R, et al. Percutaneous recanalization of chronically occluded coronary arteries: a consensus document: part I. Circulation 2005;112:2364-72.

22. Alberti KG, Zimmet PZ. Definition, diagnosis and 
classification of diabetes mellitus and its complications. Part 1: diagnosis and classification of diabetes mellitus provisional report of a WHO consultation. Diabet Med 1998; 15:539-53.

23. Di Mario C, Werner GS, Sianos G, et al. European perspective in the recanalisation of Chronic Total Occlusions (CTO): consensus document from the EuroCTO Club. EuroIntervention 2007;3:30-43.

24. Guo L, Wang J, Ding H, et al. Long-term outcomes of medical therapy versus successful recanalisation for coronary chronic total occlusions in patients with and without type 2 diabetes mellitus. Cardiovasc Diabetol 2020;19:100.

25. Martín-Timón I, Sevillano-Collantes C, Segura-Galindo A, et al. Type 2 diabetes and cardiovascular disease: Have all risk factors the same strength? World J Diabetes 2014;5:444-70.

26. Salisbury AC, Sapontis J, Grantham JA, et al. Outcomes of Chronic Total Occlusion Percutaneous Coronary Intervention in Patients With Diabetes: Insights From the OPEN CTO Registry. JACC Cardiovasc Interv 2017;10:2174-81.

27. Sanguineti F, Garot $\mathrm{P}, \mathrm{O}^{\prime}$ Connor S, et al. Chronic total coronary occlusion treated by percutaneous coronary intervention: long-term outcome in patients with and without diabetes. EuroIntervention 2017;12:e1889-97.

28. Flores-Umanzor EJ, Cepas-Guillen PL, Caldentey G, et al. Sex-based differences in chronic total occlusion management and long-term clinical outcomes. Int $\mathrm{J}$ Cardiol 2020;319:46-51.

29. Sinnaeve PR, Steg PG, Fox KA, Van de Werf F, Montalescot G, Granger CB, et al. Association of elevated fasting glucose with increased short-term and 6-month mortality in ST-segment elevation and non-ST-segment elevation acute coronary syndromes: the Global Registry of Acute Coronary Events. Arch Intern Med 2009;169:402-9.

30. Iglesias JF, Degrauwe S, Rigamonti F, et al. Percutaneous Coronary Intervention of Chronic Total Occlusions in Patients with Diabetes Mellitus: a Treatment-Risk Paradox. Curr Cardiol Rep 2019;21:9.

31. Christopoulos G, Kandzari DE, Yeh RW, et al. Development and Validation of a Novel Scoring System for Predicting Technical Success of Chronic Total Occlusion Percutaneous Coronary Interventions: The PROGRESS CTO (Prospective Global Registry for the Study of Chronic Total Occlusion Intervention) Score. JACC Cardiovasc Interv 2016;9:1-9.

32. Ruiz-Garcia J, Teles R, Rumoroso JR, et al. Comparison between diabetic and non-diabetic patients after successful percutaneous coronary intervention for chronic total occlusions in the drug-eluting stent era. Rev Port Cardiol 2015;34:263-70.

33. Zimarino M, Ricci F, Romanello M, et al. Complete myocardial revascularization confers a larger clinical benefit when performed with state-of-the-art techniques in high-risk patients with multivessel coronary artery disease: A meta-analysis of randomized and observational studies. Catheter Cardiovasc Interv 2016;87:3-12.

34. Schwartz L, Bertolet M, Feit F, et al. Impact of completeness of revascularization on long-term cardiovascular outcomes in patients with type 2 diabetes mellitus: results from the Bypass Angioplasty Revascularization Investigation 2 Diabetes (BARI 2D). Circ Cardiovasc Interv 2012;5:166-73.

35. Toma A, Gick M, Minners J, et al. Survival after percutaneous coronary intervention for chronic total occlusion. Clin Res Cardiol 2016;105:921-9.

36. Mashaly A, Rha SW, Choi BG, et al. Impact of diabetes mellitus on 5-year clinical outcomes in patients with chronic total occlusion lesions. Coron Artery Dis 2018;29:119-26.

37. Braik N, Guedeney P, Behnes M, et al. Impact of chronic total occlusion and revascularization strategy in patients with infarct-related cardiogenic shock: A subanalysis of the culprit-shock trial. Am Heart J 2021;232:185-93.

38. Wang Q, Liu H, Ding J. Outcomes of percutaneous coronary intervention in patients with coronary chronic total occlusions with versus without type 2 diabetes mellitus: A systematic review and meta-analysis. Medicine (Baltimore) 2017;96:e8499.

39. Martinez-Parachini JR, Karatasakis A, Karmpaliotis D, et al. Impact of diabetes mellitus on acute outcomes of percutaneous coronary intervention in chronic total occlusions: insights from a US multicentre registry. Diabet Med 2017;34:558-62.

40. Kim CH, Yang JH, Park TK, et al. Revascularization vs. Medical Therapy for Coronary Chronic Total Occlusions in Patients With Chronic Kidney Disease. Circ J 2018;82:2136-42.

41. van Veelen A, Claessen BEPM, Houterman S, et al. Incidence and outcomes of chronic total occlusion percutaneous coronary intervention in the Netherlands: data from a nationwide registry. Neth Heart J 202 1;29:4-13.

42. Guo L, Ding H, Lv H, et al. Impact of Renal Function on Long-Term Clinical Outcomes in Patients With Coronary Chronic Total Occlusions: Results From an Observational 
Single-Center Cohort Study During the Last 12 Years.

Front Cardiovasc Med 2020;7:550428.

43. Chen YL, Wang R, He Y, et al. Value of evaluating the coronary collateral circulation by transluminal attenuation gradient in patients with chronic total occlusion and

related influencing factors. Zhonghua Xin Xue Guan Bing
Za Zhi 2017;45:857-61

44. Bucciarelli-Ducci C, Auger D, Di Mario C, et al. CMR Guidance for Recanalization of Coronary Chronic Total Occlusion. JACC Cardiovasc Imaging 2016;9:547-56.

(English Language Editor: J. Jones)

Cite this article as: $\mathrm{Fu} \mathrm{D,} \mathrm{Li} \mathrm{H,} \mathrm{Gao} \mathrm{T,} \mathrm{Liu} \mathrm{M,} \mathrm{Feng} \mathrm{L,} \mathrm{Li} \mathrm{C,}$ Xiao X, Jiang H, Yang P, Li X. Comparison of long-term clinical outcomes of percutaneous coronary intervention for chronic total occlusion between patients with and without diabetes mellitus: a single-center retrospective observational study. Ann Palliat Med 2021;10(9):9993-10004. doi: 10.21037/apm21-2354 